\title{
Maximum Outage Capacity in Dense Indoor Femtocell Networks with Joint Energy and Spectrum Utilization
}

\author{
Youngwook Ko and Klaus Moessner, Member, IEEE
}

\begin{abstract}
We consider a multiple femtocell deployment in a small area which shares spectrum with the underlaid macrocell. We design a joint energy and radio spectrum scheme which aims not only for co-existence with the macrocell, but also for an energy-efficient implementation of the multi-femtocells. Particularly, aggregate energy usage on dense femtocell channels is formulated taking into account the cost of both the spectrum and energy usage. We investigate an energy-and-spectral efficient approach to balance between the two costs by varying the number of active sub-channels and their energy. The proposed scheme is addressed by deriving closed-form expressions for the interference towards the macrocell and the outage capacity. Analytically, discrete regions under which the most promising outage capacity is achieved by the same size of active subchannels are introduced. Through a joint optimization of the sub-channels and their energy, properties can be found for the maximum outage capacity under realistic constraints. Using asymptotic and numerical analysis, it can be noticed that in a dense femtocell deployment, the optimum utilization of the energy and the spectrum to maximize the outage capacity converges towards a round-robin scheduling approach for a very small outage threshold. This is the inverse of the traditional greedy approach.
\end{abstract}

Index Terms-Energy distribution, dense femtocell deployment, interference, opportunistic transmission, outage capacity.

\section{INTRODUCTION}

$\mathbf{F}$ UTURE cellular systems expect to comprise of dense small-cell deployments within a certain geographical service area. This is driven by dramatically increasing demands for massive data rates provided within limited spectral resources. Femtocell technology, as a promising solution towards this massive data rate wireless service, has received increasing attention in recent years [1], [2]. A femtocell being effectively a small-cost and low-power wireless access point (AP) is designed to be installed by the end-user and the femtocell(s) deployment provides indoor coverage to users in small areas such as a home or enterprise environment. Due to the short distance between transmitter and receiver in a femtocell, femtocell users benefit from better (indoor) quality of service as well as from low transmit power levels.

Manuscript received December 2, 2011; revised June 11 and August 21, 2012; accepted August 29, 2012. The associate editor coordinating the review of this paper and approving it for publication was M. Cenk Gursoy.

The authors are with the Centre for Communication Systems Research, Department of Electronic Engineering, University of Surrey, Guildford, Surrey, GU2 7XH UK (e-mail: \{y.ko, k.moessner\}@ surrey.ac.uk).
One of the main challenges for femtocell technology is how to allocate the spectrum between macro and femtocells. A femtocell can share the spectrum with the existing macrocell or can be allocated to a dedicated piece of spectrum by spectrum sharing or spectrum splitting, respectively [3]. In spectrum sharing, [4]-[6] show a higher performance by enforcing higher spatial reuse via small femtocells. Spectrum splitting, see [7], [8], between a large macrocell and small microcells is practical and benefits from lower outage probability which comes at the cost of truncation losses. However, the spectrum splitting solution is less efficient than spectrum sharing from the operator's perspective, and this solution is not always possible as the operators do not hold additional spectrum for femtocells. Therefore, we focus on spectrum sharing where femtocells are overlaid to the macrocell, the deployment is referred to as co-channel femtocells hereinafter.

Due to the fact that femtocells are randomly deployed by the end-user, it is highly likely that unexpectable interference to the macrocell service can occur (e.g., see 3GPP's LTE [9], [10] and references therein). Thus, defining a viable interference avoidance strategy is most challenging [11]-[18]. In [11], [12], the benefits of coordinating nearby femtocells in indoor deployments have been addressed, this will help to avoid, or at least limit interference. In [13], a roadmap for interference avoidance in femtocell deployments was provided with an emphasis on utilizing self-organization techniques. In [14], a distributed utility-based signal to interference and noise ratio (SINR) adaptation at femtocells was proposed aiming alleviate the interference (caused by the femtocells) at the macrocell. In [15], a technique of adjusting femtocell coverage was proposed to minimize cross-tier interference while [16] addressed a method of power control for pilot and data that ensures a constant femtocell radius in the downlink with a low impact to the macrocell. The authors in [17] analyzed the outage performance of a femtocell network with respect to the co-channel interference between the macrocell and the femtocell. In [18], adjusting the maximum transmit power of femtocell users was proposed to manage cross-tier interference. Furthermore, as a result of the dense femtocell deployment, the sum power usage of the system increases and this causes high interference towards the incumbent receiver. With regards to the interference requirement, it is desired to decrease the sum power usage at the cost of system capacity of the femtocells. The energy efficient solution is important to resolve the former while the spectral efficient solution is needed to overcome the latter. In this context, one major 
challenge is to investigate the sum energy usage by the dense femtocells and its impact on the system capacity. As per the energy usage of the femtocell networks, only very few preliminary results are available [19], [20], and no concern was given to the joint energy and spectrum utilization between nearby femtocells.

In this work, we consider orthogonal frequency division multiplexing access (OFDMA) based femtocells, distributed in indoor deployment and overlaid to the existing macrocell by spectrum sharing. For given sub-channels available at the femtocells, we propose a joint energy and spectrum resource (i.e., sub-channels) utilization approach for indoor dense femtocell networks. This aims not only for the co-existence with the conventional macrocell, but also for an energy-aware implementation of multi-femtocells deployment. To this end, aggregate energy usage among femtocells is designed. Particularly, we provide methods of (i) mathematically formulating the aggregate amount of the energy usage by taking into account the cost of both the sub-channels at the femtocells and individual femtocell energy usage for the control and data transmissions, (ii) finding the maximum amount of the energy usage per femtocell allowing the aggregate interference (by the nearby femtocells) at the incumbent macrocell receiver below the pre-defined level, and (iii) self-organizing the energy usage to balance between the two costs by varying the energy per sub-channel as well as the number of active sub-channels per femtocell in a distributed manner. From an information theoretic perspective, characterization of the maximum achievable rates of the indoor dense femtocells downlink fading channels is provided at the allowance of the outage in a rate. In particular, the performance of the proposed scheme is analyzed by deriving expressions for the aggregate energy usage, the resulting interference toward the macrocell receiver and the outage capacity with limited energy usage at the femtocells. We also formulate the optimization problem in which the achievable outage capacity per femtocell is maximized under realistic constraints by finding, jointly, the optimum energy usage as well as the optimum number of active sub-channels per femtocell. As per our asymptotic analysis and numerical results, it will be clearly found that in a dense femtocell downlink fading channels deployment, the optimum energy-utilizing scheduling in the sense of the maximization of the outage capacity per femtocell converges towards the round-robin scheduling.

The paper is organized as follows. The system model is introduced and the problem is described formally in Sections II and III, respectively. In Section IV, the aggregate energy usage for indoor multiple femtocells for the control and the data transmissions is modeled. In Section V, the system performance measures, such as the interference and the outage capacity are derived, followed by the mathematical investigations of their dependence on the joint energy and spectrum resource utilization. Section VI contains the optimization solution toward the maximum outage capacity in the femtocell deployment by exploiting the proposed aggregate energy usage model under the co-existence with the macrocell network, while Section VII provides further asymptotic analysis for several cases. Section VIII presents numerical results and is followed by conclusions in Section IX.

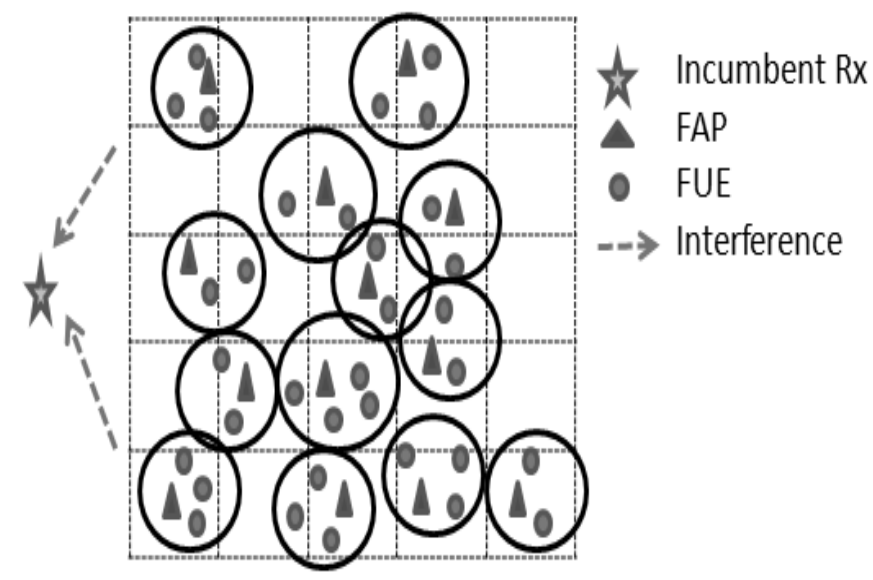

Fig. 1. Multiple orthogonal frequency division multiplexing access (OFDMA) femtocells deployment layout in a small region such as a $5 \times 5$ grid layout of the enterprise environment at the presence of a nearby incumbent receiver.

\section{SySTEM MODEL}

As shown in Fig. 1, we consider $L$ femtocells, each of which serves $N_{l}$ femtocell user equipments (FUEs) in a radius $\left(r^{f_{l}}\right)$ and, for the downlink, $L$ femtocells cognitively access the radio spectrum, licensed to the underlaid macrocell network. $L$ femtocells are randomly distributed in a small area (e.g., enterprise environment) on the coverage of the macrocell network supporting a radius $\left(r^{m}\right)$ and co-channel deployment of the femtocells causes interference toward macrocell UEs located near at the femtocells. Let each femtocell coordinate to each other and orthogonally operate over the available incumbent channels in the frequency domain while avoiding the co-tier interference among neighboring femtocells.

For the downlink of each femtocell, we employ orthogonal frequency division multiplexing (OFDM) (e.g., 3GPP's LTE [21]). Particularly, let each FUE in femtocell $l$ be allocated to $\bar{n}$ sub-channels (or, equivalently, sub-carriers) for given $m$ incumbent radio channels, where it is assumed that $m$ channels are originally licensed to a macrocell UE near the femtocells and for the simple analysis and without loss of the generality, the value of $\bar{n}$ is given such that $\bar{n}=m / \sum_{l} N_{l}$ is an integer hereafter. $^{1}$

Suppose that the sub-channels between FUEs and the $l$ th femtocell access point (FAP $l$ ), $\forall l$ are independent and Rayleigh flat fading and they are known perfectly at the receiver. Therefore, the received signal to the interference and noise ratio (SINR) at sub-channel $i$ for $i=1, \cdots, \bar{n} N_{l}$ can be represented in femtocell $l$ as

$$
\rho_{l i}=\frac{g_{l i} P_{d}^{l i}}{I+\sigma^{2}} \quad \text { for } i=1, \cdots, \bar{n} N_{l}
$$

where $g_{l i}=x_{l i} \Theta_{f_{l}} d_{l i}^{-L_{f}}$ denotes a channel gain between the transmitter and the receiver on sub-channel $i, \Theta_{f_{l}}$ is a $\log -$ normal random variable that represents the shadowing effect

\footnotetext{
${ }^{1}$ Notice that in practice, $\bar{n}$ is an integer nearest to a real value of the ratio, $m / \sum_{l} N_{l}$, toward zero. Also, note that the number $m$ of channels licensed to the macrocell user can be obtained by referring to the broadcasted information from the macrocell base station or via spectrum sensing (for details, refer to [22], [23] and references therein.)
} 
on a femtocell environment, $d_{l i}$ is the distance between the transmitter and the receiver, $L_{f}$ is a path-loss exponent in the femtocell and $x_{l i}$ stands for the post-processing gain of the Rayleigh fading channel coefficients and is a Chi-square distributed random variable with $2 k$ degrees of freedom (i.e., $x_{l i} \sim \chi_{2 k}^{2}$ ) where $k$ denotes the number of receive antennas along with multi-antenna techniques (e.g., maximal ratio combining). Notice in (1) that $P_{d}^{l i}$ is the data transmit power, $I$ denotes the cross-tier interference on average received from the macrocell base station (MBS) at each FUE [3], [4] and $\sigma^{2}$ is the variance of the complex-valued zero-mean additive white Gaussian noise (AWGN).

On every data transmission, we are aware of the energy usage at two planes inherent in the networked femtocells: (i) the control plane, and (ii) the data plane. In the control plane, firstly, exchange of control signaling between FAPs and FUEs happens. In particular, for given $\bar{n}$ sub-channels per FUE, the FAP $l$ randomly activates only a subset of $n_{l}(\leq \bar{n})$ subchannels per FUE according to the Uniform distribution at every time slot. This enables that each entry (i.e., each subchannel) in the subset is equally likely activated and this random selection at each time slot produces fairness among users in terms of equal probability of accessing the channel. Then, among $N_{l}$ FUEs at femtocell $l, N_{l} n_{l}$ sub-channels become active for scheduling in a given time slot. Only on such $N_{l} n_{l}$ sub-channels randomly activated, therefore, the exchange of signaling occurs for the purpose of radio resource scheduling. Let the power allocation level of $P_{c}^{l i}=P_{c} \psi_{l i}$ for the signaling be assigned to each active sub-channel $i, \forall i$. Here, $\psi_{l i}$ stands for the propagation loss compensation component so that the desired power level $P_{c}$ at the receiver is achieved on average. Secondly, for given such $N_{l} n_{l}$ active sub-channels, each femtocell employs the opportunistic radio resource scheduling scheme, from the system perspective this is seen as being optimal in the sense of the maximum achievable rate [24][26]. That is, in the data plane, allocating all transmit power to only the best among $N_{l} n_{l}$ active sub-channels is motivated for the data transmission at femtocell $l, \forall l$ at each time slot. The criterion of selecting the best sub-channel is to select the best whose SINR is defined as $\rho_{l} \triangleq \max _{i} \rho_{l i}$. Let the data transmission by the chosen best is made at the power $P_{d}^{l i}$ in (1), where $P_{d}^{l i}=P_{d} \psi_{l i}, \forall i$ and $P_{d}$ is the desired receiving power for the data, similar to $P_{c}$. Therefore, the resulting sum rate at femtocell $l$ for $l \in\{1, \cdots, L\}$ can be given by

$$
C_{l}=\log _{2}\left(1+\rho_{l}\right)=\log _{2}\left(1+\bar{\rho}_{l} x_{l} P_{d}\right)
$$

where $x_{l}=\max _{i} x_{l i}$ and $\bar{\rho}_{l}$ denotes the average normalized SINR, i.e., $\bar{\rho}_{l}=\Theta_{f_{l}} /\left(I+\sigma^{2}\right)$, for a given noise as the sum of $\sigma^{2}$ and $I$.

\section{PROBLEM Formulation}

Consider the case of using the outage-sensitive and the energy-limited femtocells application. In this case, we focus on maximizing the outage capacity $C_{\text {out }, l}$ per femtocell. The outage capacity used in this work is referred to as the maximum achievable rate $R$ such that the probability that the sum rate $\left(C_{l}\right)$ per femtocell is less than or equal to the rate $(R)$ is less than or equal to a given threshold $\epsilon$ for $\epsilon>0$ (i.e., $\left.\operatorname{Pr}\left(C_{l} \leq C_{\text {out }, l}\right) \leq \epsilon\right)$.
To this end, we account for optimal selection cost (i.e., optimal number) of active sub-channels per FUE and their power allocation levels. Meanwhile, the sum energy usage $\left(E_{l}\right)$ on the total available sub-channels per femtocell is limited to a target level $E_{o, l}$ (i.e., $E_{l}=E_{o, l}$ ) and the resulting interference $\left(I^{f m}\right)$ by the use of $E_{l}$ from femtocell $l, \forall l$ toward the incumbent receiver remains equal to the given threshold $I_{o}$. Therefore, the problem at each femtocell can be posed for all $l$ as

$$
\begin{gathered}
\max \quad C_{\text {out }, l}, \\
\text { s.t. } \quad \operatorname{Pr}\left(C_{l} \leq C_{\text {out }, l}\right) \leq \epsilon, \\
E_{l} \leq E_{o, l}, \\
I^{\text {fm }} \leq I_{o} .
\end{gathered}
$$

To resolve the problem in (3)-(6), we notice the fact that there exists a tradeoff between the energy usage by femtocells and the resulting interference to the macrocell UEs, where the higher the energy usage at femtocells, the stronger the interference to the victim macrocell UEs is. Therefore, our main idea is to find out an appropriate way of adjusting the energy usage by femtocells such that this energy usage is traded for an acceptable level of the interference (i.e., $I^{f m} \leq I_{o}$ ). In particular, we first investigate how the energy usage by femtocells and $I^{f m}$ behave in terms of the power allocation levels (i.e., $P_{c}$ and $P_{d}$ ) on the control and the data planes. Here, selecting the amount $E_{l}$ of the energy usage per femtocell subject to the coexistence constraint (6) is taken into account while allowing coordination among femtocells (and this coordination in our framework will be based on simply referring to static context information such as, for example, the number of available FUEs at each femtocell.)

In addition, based on the properly chosen energy budget $E_{o, l}$, the goal is to balance the energy usage between the control and the data planes at each femtocell by taking into account an optimal selection of the number of active subchannels per FUE and thereby the maximization of $C_{\text {out }, l}$. This energy usage balance is performed in a distributed fashion among femtocells.

\section{Aggregate Energy Usage by Networked FEMTOCELLS}

We address the aggregate energy usage between all the FUEs and the networked femtocells at both the control and the data planes from the signal processing perspective. For simplicity in analysis and without loss of generality, we consider hereafter the worst case interference, where all the FUEs are located at the cell-edge of each femtocell and thus the resulting downlink energy usage per femtocell is largest. This leads to an asymptotic situation where the interference $I^{f m}$ caused by the femtocells results in highest.

For the mathematical treatment of the energy usage between FUEs and femtocells, we first refer to the energy usage by multiple users in a typical multipoint-to-point communications [27]. As per the energy usage in [27], we can formulate that the energy usage at each femtocell, comprising multiple femtocell UEs, is decomposed into two terms; one term for energy usage at the control plane and the other at the data plane. In particular, it can be obtained that the sum energy 
$E_{l}$ at femtocell $l$ for a given time period $T_{f}$ is represented when all $\bar{n}$ sub-channels available at each FUE are active (i.e., $n_{l}=\bar{n}$ ) as

$$
E_{l}=N_{l} \bar{n} \psi_{l} P_{c} T_{f}+\psi_{l} \tilde{P}_{d} T_{f}, \quad \forall l
$$

where we have the propagation-loss compensation $\psi_{l}=\psi_{l, i}$ due to equally likely the same distance between FUEs and the FAP $l$ as the radius $r^{f_{l}}$ and thereby, $\psi_{l}=\left(r^{f_{l}}\right)^{L_{f}}$, and $\tilde{P}_{d}$ stands for the desired power $P_{d}$ only when $n_{l}=\bar{n}$. In (7), let $\tilde{P}_{d}=\eta^{-1} P_{c}$ also be for a given ratio $\eta$ of the power allocations between the control and the data planes when $n_{l}=$ $\bar{n}$.

Using this ratio $\eta$ and (7), the aggregate sum energy $\left(E_{a}\right)$ at the networked femtocells can be achieved by the sum up of $E_{l}$ for all $l$ and this is represented when $n_{l}=\bar{n}$ as

$$
E_{a}=\sum_{l=1}^{L}\left(N_{l} \bar{n} P_{c} \psi_{l} T_{f}+\eta^{-1} P_{c} \psi_{l} T_{f}\right) .
$$

Notice in (8) that the first term corresponds to the energy usage in the control plane while the second term in the data plane.

Now, let us consider the case when $n_{l}<\bar{n}$. Similar to (7), then, $E_{l}$ in this case can be represented as

$$
E_{l}=N_{l} n_{l} P_{c} \psi_{l} T_{f}+P_{d} \psi_{l} T_{f}, \quad \forall l .
$$

From (8) and (9), it is straightforward to present the expression of $E_{a}$ when $n_{l}<\bar{n}$ as

$$
E_{a}=\sum_{l=1}^{L}\left(N_{l} n_{l} P_{c} \psi_{l} T_{f}+P_{d} \psi_{l} T_{f}\right)
$$

where $P_{d}$ denotes the desired power allocation for the data at the receiver when $n_{l}<\bar{n}$.

Regardless of the values of $n_{l}$, when maintaining the sum energy per femtocell constant, the power allocation $P_{d}$ in (10) can be now presented in a closed-form expression in terms of $n_{l}$ and $P_{c}$. Particularly, treat $E_{l}$ in the case when $n_{l}=\bar{n}$ as its energy budget (i.e., $E_{o, l}=E_{l}$ in (7)). Then, when $n_{l}<\bar{n}$, let $E_{l}$ in (9) be bounded by $E_{o, l}$. By limiting (9) to (7) and after simple mathematical manipulations, therefore, we can achieve that $P_{d}$ in (10) is given by

$$
\begin{aligned}
P_{d} & \leq\left(\frac{E_{o, l}}{\psi_{l} T_{f}}-N_{l} n_{l} P_{c}\right) \\
& =N_{l}\left(\bar{n}-n_{l}\right) P_{c}+\tilde{P}_{d}, \quad \forall l
\end{aligned}
$$

so that the requirement of $E_{l} \leq E_{o, l}$ in (5) is satisfied what ever $n_{l} \leq \bar{n}$ is.

From (8)-(12), we can find that when $n_{l} \leq \bar{n}, \forall l$, the aggregate sum energy usage by the networked femtocells is represented as a function of $n_{l}$ and $P_{c}$. Specifically, this aggregate energy usage on the control plane is represented for $n_{l} \leq \bar{n}$ as

$$
\sum_{l=1}^{L} N_{l} n_{l} \psi_{l} P_{c} T_{f}
$$

while the aggregate energy usage on the data plane is for $n_{l} \leq \bar{n}$

$$
\sum_{l=1}^{L} N_{l}\left(\bar{n}-n_{l}+\eta^{-1}\right) \psi_{l} P_{c} T_{f}
$$

It can be seen from (13)-(14) that as $P_{c}$ increases for a given $n_{l}, \forall l$, the aggregate energy usage at both the control and the data planes increases linearly and thereby, the larger amount $E_{a}$.

Moreover, it is worth pointing out from (13)-(14) that for a properly chosen $P_{c}$, the selections of $n_{l}, \forall l$ influence the energy usage balance between the control and the data planes at each femtocell. In the subsequent section, decision on such energy usage balance through distributed selection of $n_{l}$ will be taken into account to analyze the outage capacity at each femtocell, following details on selection criterion of $P_{c}$ based on the interference requirement.

\section{INTERFERENCE AND OUTAGE CAPACITY ANALYSIS}

\section{A. Cross-tier interference toward the incumbent receiver}

To guarantee the interference requirement in (6), we study a selection criterion of the power allocation $P_{c}$ by making use of the energy usage in (13) and (14).

The amount of the energy usage in (13) and (14) is related to the interference $I^{f m}$ at the incumbent receiver for given distances (denoted by $d_{l}, \forall l$ ) between the femtocells and the incumbent receiver. ${ }^{2}$ Due to the path-loss between femtocells and the incumbent receiver, this interference can be written by

$$
I^{f m}=\sum_{l=1}^{L} N_{l} \bar{n} P_{c} \psi_{l} d_{l}^{-L_{f}}+\eta^{-1} P_{c} \psi_{l} d_{l}^{-L_{f}}
$$

where $d_{l}^{-L_{f}}$ stands for the path-loss between femtocell $l$ and the incumbent receiver.

From (15), when having $I^{f m} \leq I_{o}$, we can have that $P_{c}$ should satisfy

$$
(15) \leq I_{o} \Rightarrow P_{c} \leq \frac{I_{o}}{\sum_{l=1}^{L} N_{l} \bar{n} \psi_{l} d_{l}^{-L_{f}}+\eta^{-1} \psi_{l} d_{l}^{-L_{f}}} .
$$

Recalling that $\psi_{l}=\left(r^{f_{l}}\right)^{L_{f}}$ in (7) and inserting this into (16), therefore, it can be found that $P_{c}$ in (16) is further expressed as

$$
P_{c} \leq \frac{I_{o}}{\sum_{l=1}^{L} N_{l} \bar{n}\left(\frac{d_{l}}{r^{f_{l}}}\right)^{-L_{f}}+\eta^{-1}\left(\frac{d_{l}}{r^{f_{l}}}\right)^{-L_{f}}} .
$$

It can be observed from (17) that for given $L$ and $N_{l}, \forall l$, $P_{c}$ satisfying $I^{f m} \leq I_{o}$ increases exponentially with $d_{l}$. Also, it can be found from this equation that as the number of $L$ femtocells increases, $P_{c}$ should decrease while guaranteeing $I^{f m} \leq I_{o}$. With regard to $\eta$, as shown in (17), $P_{c}$ can further scale inversely as $\eta$ decreases.

\section{B. Outage capacity of femtocell-of-interest}

When $P_{c}$ satisfying the equality in (17) is used in (12), $P_{d}$ in a given $E_{l}$ can be given when $n_{l} \leq \bar{n}$ by

$$
P_{d}=N_{l}\left(\bar{n}-n_{l}+\eta^{-1}\right) \frac{I_{o}}{\sum_{l=1}^{L} N_{l} \bar{n}\left(\frac{d_{l}}{r^{f_{l}}}\right)^{-L_{f}}+\eta^{-1}\left(\frac{d_{l}}{r^{f_{l}}}\right)^{-L_{f}}} .
$$

\footnotetext{
${ }^{2}$ This distance $d_{l}$ can be known at the femtocells by taking into account the path-loss between the femtocells and the incumbent receiver while listening to reference signals (e.g., channel quality indicator (CQI) feedbacks [28], busy bursts transmission [29]) sent by the incumbent receiver to the MBS.
} 
Inserting (18) into (2), the sum capacity per femtocell in (2) can be represented as

$$
C_{l}=\log _{2}\left(1+\bar{\rho}_{l} \frac{x_{l} N_{l}\left(\bar{n}-n_{l}+\eta^{-1}\right) I_{o}}{\sum_{l=1}^{L} N_{l} \bar{n}\left(\frac{d_{l}}{r^{f_{l}}}\right)^{-L_{f}}+\eta^{-1}\left(\frac{d_{l}}{r^{f_{l}}}\right)^{-L_{f}}}\right) .
$$

Using (17) and (19), $\operatorname{Pr}\left(C_{l} \leq C_{\text {out }, l}\right) \leq \epsilon$ in (4) can be rewritten in a closed-form as

$$
F_{x_{l}}\left(\left(2^{C_{\text {out }, l}}-1\right)\left(\bar{\rho}_{l} P_{c}\left(N_{l}\left(\bar{n}-n_{l}\right)+\eta^{-1}\right)\right)^{-1}\right) \leq \epsilon
$$

where $F_{x_{l}}(x)$ stands the cumulative distribution function (CDF) of $x_{l}$.

According to the higher order statistics and recalling $x_{l}=$ $\max _{l} x_{l i}$ in (2), this CDF can be given by $F_{x_{l}}(x)=F_{x}(x)^{N_{l} n_{l}}$ where the subscript $x$ in $F_{x}(\cdot)$ stands for $x_{l i}, \forall i$ (and thus, $\left.x \sim \chi_{2 k}^{2}\right)$ and its CDF is $F_{x}(x)=\gamma(k, x) / \Gamma(k)$. Here, $\gamma(\cdot, \cdot)$ is the incomplete gamma function, and $\Gamma(\cdot)$ is the complete gamma function [30].

As per this, to achieve a closed-form expression for $C_{o u t, l}$ satisfying the inequality in (20), an inverse of $\gamma(\cdot, \cdot)$ is needed but it is not trivial. Thus, we provide an asymptotic and accurate closed-form expression for (20). To this end, it is referred to some of the well-known results on the asymptotic behavior of the maximum among independent random variables in our work. Let us define $y \triangleq\left(x_{l}-a_{m_{l}}\right) / b_{m_{l}}$, where the parameter $m_{l}=N_{l} n_{l}$ is hereinafter used for simple description, $a_{m_{l}}=$ $F_{x}^{-1}\left(1-1 / N_{l} n_{l}\right)$, and $b_{m_{l}}=F_{x}^{-1}\left(1-1 / N_{l} n_{l} \mathrm{e}\right)-a_{m_{l}}$. Then, when $m_{l}=N_{l} n_{l}$ is large (e.g., $N_{l} n_{l} \geq 10$ ), the distribution of $y$ converges to Gumbel distribution [31] and its CDF is as

$$
G_{y}(y)=\exp (-\exp (-y))
$$

From (21) and (20), the CDF of $x_{l}$ can be rewritten in terms of the CDF of $y$ and thus, $\operatorname{Pr}\left(C_{l} \leq C_{\text {out }, l}\right) \leq \epsilon$ in (20) can be represented in terms of the distribution of $y$ as

$G_{y}\left(\frac{\left(2^{C_{\text {out }, l}}-1\right)\left(\bar{\rho}_{l} P_{c}\left(N_{l}\left(\bar{n}-n_{l}\right)+\eta^{-1}\right)\right)^{-1}-a_{m_{l}}}{b_{m_{l}}}\right) \leq \epsilon$

Here, for details of $a_{m_{l}}$ and $b_{m_{l}}$ with respect to $N_{l} n_{l}$, we further introduce following proposition.

Proposition 1: For large values of $m_{l}=N_{l} n_{l}$, we have

$$
\begin{aligned}
a_{m_{l}} & =\log \frac{m_{l}}{\Gamma(k)}+\log \left(\log m_{l}+(k-1) \log \log m_{l}\right)^{(k-1)} \\
& +\mathrm{o}\left(\log \log \log m_{l}\right) \\
b_{m_{l}} & =1+\log \left(1+\left(\log m_{l}+(k-1) \log \log m_{l}\right)^{-1}\right)^{(k-1)} \\
& +\mathrm{o}\left(\log \log \log m_{l}\right)
\end{aligned}
$$

where $\mathrm{o}(\cdot)$ is a little o notation, i.e., $g(x)=\mathrm{o}(f(x))$ means that $\lim _{x \rightarrow \infty} g(x) / f(x)=0$ [30].

Proof: See Appendix.

Using this proposition and (22), we provide the following proposition.

Proposition 2: When $N_{l} n_{l}$ is large for given $\bar{n}, L, \epsilon$, and $n_{l} \leq \bar{n}$, the outage capacity, $C_{\text {out }, l}$, at femtocell $l$ can be

$\log _{2}\left(1+\bar{\rho}_{l} P_{c}\left(N_{l}\left(\bar{n}-n_{l}\right)+\eta^{-1}\right)\left(a_{m_{l}}-b_{m_{l}} \log (-\log \epsilon)\right)\right)$ while maintaining the requirements (4)-(6), where recall that $P_{c}$ is given in (17) by $P_{c}=I_{o} / \sum_{l=1}^{L}\left(N_{l} \bar{n}+\eta^{-1}\right)\left(\frac{d_{l}}{r^{f_{l}}}\right)^{-L_{f}}$. Proof: See Appendix.

It can be found from (25) that for given $N_{l}, n_{l}, \epsilon$ and $\eta$, the outage capacity is increasing in the logarithmic scale with respect to $P_{c}$. Recall that as per (17), this allocation power of $P_{c}$ in (25) decreases with the number of $L$ femtocells due to the interference requirement. This observation from (25) can therefore reveal that the outage capacity in (25) decreases as the number $L$ of networked femtocells increases for a fixed $n_{l}$.

Also, for given $P_{c}$ and $\bar{n}$, it can be observed from (25) that the term $\left(N_{l}\left(\bar{n}-n_{l}\right)+\eta^{-1}\right)$ linearly decreases with $n_{l}$ while the term $\left(a_{m_{l}}-b_{m_{l}} \log (-\log \epsilon)\right)$ increases with $n_{l}$ in the logarithmic scale. Therefore, the former term can be traded off for the latter by increasing the size of $n_{l}$. This reveals that there exist opportunities of enhancing (25) by a proper selection of the size of $n_{l}$. Criterion of selecting optimal size of $n_{l}$ will be addressed in the following section.

\section{Optimal SOLUTION}

Making use of the trade-off terms on the outage capacity addressed in (25) the outage capacity maximization problem stated in (3) is solved in this section. Notice that while satisfying the requirements (4)-(6), it turns out that the outage capacity expression in (25) is a function of $n_{l}$ for given system parameters. Therefore, the optimization problem in (3) can be rewritten as

$$
\begin{gathered}
\max _{n_{l} \in\{1, \cdots, \bar{n}\}} \log _{2}\left(1+\bar{\rho}_{l} P_{c}\left(N_{l}\left(\bar{n}-n_{l}\right)+\eta^{-1}\right)\right. \\
\left.\times\left(a_{m_{l}}-b_{m_{l}} \log (-\log \epsilon)\right)\right) .
\end{gathered}
$$

This problem is a concave optimization problem since the second derivative of the outage capacity in terms of $n_{l}$ is not positive, i.e., $\partial^{2} C_{\text {out }, l} / \partial^{2} n_{l} \leq 0$. To mathematically solve this problem, a Lagrange multiplier method can be applied. Via this method, it turns out that the optimal solution in our case can be simplified to find the value minimizing the first derivation of the outage capacity in terms of $n_{l}$. The resulting optimal selection of $n_{l}$ can be formulated for a given $\epsilon$ as

$$
n_{l}^{o p t}=\arg \min _{n_{l}}\left|\mathcal{A}_{1}\left(n_{l}, \epsilon\right)-\mathcal{A}_{2}\left(n_{l}, \epsilon\right)\right|
$$

where we have

$$
\begin{aligned}
\mathcal{A}_{1}\left(n_{l}, \epsilon\right) & =N_{l} \log \left(\frac{N_{l} n_{l}}{\Gamma(k)}\left(\log N_{l} n_{l}\right)^{k-1}\right)-N_{l} \log (-\log \epsilon) \\
& \times\left(1+(k-1) \log \left(1+\left(\log N_{l} n_{l}\right)^{-1}\right)\right) \\
\mathcal{A}_{2}\left(n_{l}, \epsilon\right) & =\left(N_{l}\left(\bar{n} n_{l}^{-1}-1\right)+\eta^{-1} n_{l}^{-1}\right) \\
& \times\left(1+\frac{k-1}{\log N_{l} n_{l}}+\frac{\log (-\log \epsilon)(k-1)}{\left(1+\log N_{l} n_{l}\right) \log N_{l} n_{l}}\right) .
\end{aligned}
$$

Here, it is worth pointing out from (27) that the optimum $n_{l}^{\text {opt }}$ maximizing the outage capacity relies on $\epsilon$ and is independent of the interference threshold (i.e., $I_{o}$ ). Intuitively, this is because the optimum $n_{l}^{\text {opt }}$ intends to optimally balance the energy usage trade-off between the control and the data planes in $(25)$ and at both the planes, the overall energy consumption limited by the interference requirement is controlled by the selection of $P_{c}$ in (17). 
In the case when $k=1$, for example, the optimum $n_{l}^{o p t}$ in (27) can be simplified to

$$
\arg \min _{n_{l}}\left|\log \frac{N_{l}}{-\log \epsilon}+1+\left(\log n_{l}-n_{l}^{-1}\left(\bar{n}+\left(\eta N_{l}\right)^{-1}\right)\right)\right| .
$$

Notice that when $k=1$, (28) can also be achievable by using the inverse of the simplified $F_{x}(x)$ (i.e., $F_{x}(x)=1-\exp (-x)$ ) without $G_{y}(y)$ in (22). The corresponding expression for $n_{l}^{o p t}$ is still equivalent to $n_{l}^{o p t}$ in (28).

As per (28), it can be observed that when $k=1$, the optimum $n_{l}^{o p t}$ depends on $\epsilon$ for given $N_{l}, \eta$ and $\bar{n}$. Particularly, notice the fact from (28) that for a given $\epsilon$, the argument of $|(\cdot)|$ can be shown to be a monotonically increasing function of $n_{l}$ since $\partial(\cdot) / \partial n_{l}>0$ while (.) being strictly concave (i.e., $\left.\partial^{2}(\cdot) / \partial^{2} n_{l}<0\right)$. Then, for given values of $\epsilon$ producing the term $\left(\log N_{l}-\log (-\log \epsilon)\right)$ in (28) negative, it turns out that the optimum $n_{l}^{\text {opt }}$, equating the argument $(\cdot)$ closest to the zero, is an intermediate value between 1 and $\bar{n}$. Specifically, this means that as far as $\epsilon \in\left(0, \mathrm{e}^{\left(-N_{l} \cdot \text { e) }\right.}\right]$ (e.g., $\epsilon \leq 0.07$ when $N_{l}=1$ ), the value of the optimum $n_{l}^{\text {opt }}$ between 1 and $\bar{n}$ can be found. In addition, it can be found from (28) that for given $N_{l}$ and $\bar{n}$, less $\epsilon$ (while $\epsilon \geq \mathrm{e}^{\left(-N_{l} \cdot \mathrm{e}\right)}$ ), closer the term $\log (-\log \epsilon)$ is to $N_{l}$. Therefore, this reveals that when $\epsilon \geq \mathrm{e}^{\left(-N_{l} \cdot \mathrm{e}\right)}$, the smallest among candidate $n_{l}$ 's is the optimum $n_{l}^{\text {opt }}$ accordingly.

With regard to $\eta$, it is worth pointing out from (28) that for very small $\eta$, the argument of $|\cdot|$ in (28) is dominated by the term having $\left(\eta N_{l}\right)^{-1}$. Thus, it can be stated that for very small $\eta$, we asymptotically have $n_{l}^{o p t}=\arg \min _{n_{l}} n_{l}^{-1}(\bar{n}+$ $\left.\left(\eta N_{l}\right)^{-1}\right)$. This reveals that for very small $\eta$, the largest possible value of $n_{l}$ is the optimal.

\section{Asymptotic Results}

In this section, we consider two extreme cases: i) case when the number $\bar{n}$ of sub-channels available goes to infinity; ii) case when the outage threshold $\epsilon$ goes to zero. Accordingly, we investigate how the achievable optimum number of active sub-channels behaves in the two extreme cases.

\section{A. Case when $\bar{n}$ is large}

Let the size $n_{l}$ of active sub-channels be $n_{l}=\beta \bar{n}$ for a fixed ratio $\beta \in[1 / \bar{n}, 1], \forall l$. This means that when $\bar{n}$ increases, $n_{l}$ also does but the ratio of $n_{l}$ to $\bar{n}$ is fixed to $\beta$. As $\bar{n}$ goes to infinity such that $n_{l}=\beta \bar{n}$ is much greater than $k$ (i.e., $\beta \bar{n} \gg k$ ) for given $k$ and $\beta$, we can have from (23) and (24):

$$
\begin{aligned}
& a_{m_{l}} \cong \log N_{l} \beta \bar{n}+(k-1) \log \log N_{l} \beta \bar{n} \\
& b_{m_{l}} \cong 1 .
\end{aligned}
$$

When inserting these asymptotic observations into (25), the outage capacity for very large $\bar{n}$ can be given for all $l$ by

$$
\begin{aligned}
C_{\text {out }, l} & \cong \log \bar{\rho}_{l} P_{c}+\log N_{l} \bar{n}(1-\beta) \\
& =\log \frac{N_{l}(1-\beta) I_{o}}{\sum_{l=1}^{L} N_{l}\left(\frac{d_{l}}{r^{f_{l}}}\right)^{-L_{f}}}+\log \bar{\rho}_{l}
\end{aligned}
$$

where recall that $P_{c}$ in (17) is defined as a function of $\bar{n}$ as well as the given interference threshold $I_{o}$. It can be clearly seen from (31) that for large $\bar{n}, C_{\text {out }, l}$ turns out to scale only with $\beta$ for given $I_{o}, N_{l}$ and $\bar{\rho}_{l}$.

Interestingly, it is worth pointing out from (31) that for large $\bar{n}$, the optimum ratio $\beta$ maximizing the outage capacity can be found to be the smallest possible value of $\beta$. This means that for large $\bar{n}$, the optimum size of active subchannels should be one in order to maximize the outage capacity. Therefore, in such extreme case, the interferenceaware round-robin scheduling among available sub-channels would be optimal in the sense of maximization of the outage capacity at femtocells.

The intention behind this is as follows. Due to the channel hardening effect [32], the incremental scheduling gain in (25) becomes negligible for very large $\bar{n}$. In particular, it can be observed from (29)-(30) that for very large $\bar{n}$, the incremental values of $a_{m_{l}}$ and $b_{m_{l}}$ are negligible and zero, respectively. This decreases the benefits from the scheduling at very large $\bar{n}$. Thus, we can obtain that for very large $\bar{n}$, the outage capacity in (31) is dominated by only the other term $P_{c} N_{l} \bar{n}(1-\beta)$. Further referring to $P_{c}$ below (25), for very large $\bar{n}$, the above observation can be achievable for the maximum outage capacity. For example, as per $\beta \in\left[\bar{n}^{-1}, 1\right]$ for $n_{l} \in\{1, . ., \bar{n}\}$ and very large $\bar{n}$, the optimal value for $\beta$ will be $\beta=\bar{n}^{-1}$. Equivalently, it is optimal to have $n_{l}=1$ (i.e., the interference-aware round-robin scheduling) for the maximum outage capacity for very large $\bar{n}$.

\section{B. Case when $\epsilon$ is very small}

In this section, let us consider $N_{l}$ single antenna (i.e., $k=1$ ) FUEs in a femtocell of interest for simple analysis and the extreme case when $\epsilon$ decreases toward zero is considered. ${ }^{3}$ By referring to (28), then, it can be obtained that for very small $\epsilon, n_{l}^{o p t}$ is expressed as (32) that is shown on the top of the next page. In this equation, $c=1+\log N_{l}$ is a constant for a given $N_{l}$.

It can be shown from (32) that as $\epsilon$ decreases (under $\epsilon \epsilon$ $\left.\left(0, \mathrm{e}^{-N_{l} \cdot \mathrm{e}}\right]\right)$, the term $\log (-\log \epsilon)$ in (32) increases and thus, candidate value for the optimum $n_{l}^{\text {opt }}$ should increase the last term $\left(\log n_{l}-n_{l}^{-1}\left(\bar{n}+\left(\eta N_{l}\right)^{-1}\right)\right)$ in (32). Notice the fact that this last term is a monotonically increasing function of $n_{l}$. This therefore reveals that for small $\epsilon \in\left(0, \mathrm{e}^{-N_{l} \cdot \mathrm{e}}\right]$, the optimum $n_{l}^{o p t}$ maximizing the outage capacity turns out to be the largest possible value among candidate $n_{l}$ 's. This is the inverse of the case that as $\epsilon\left(\geq \mathrm{e}^{-N_{l} \cdot \mathrm{e}}\right)$ decreases towards zero while $N_{l}$ grows without bound, $n_{l}^{o p t}$ is the smallest among candidate $n_{l}$ 's, as discussed in Section VI. Also, when $\epsilon=0$ (i.e., the zero outage probability), there will be no transmission ensuring the zero outage probability due to its definition (i.e., $\left.F_{x_{l}}^{-1}(\epsilon=0)=0\right)$ and thus, $C_{o u t, l}=0$.

\section{NumERICAL AND Simulation Results}

For numerical and simulation results, we consider femtocells deployment in a $5 \times 5$ grid layout of geographical environment such as, for example, enterprize environments. Here, both penetration and propagation losses are in line with

\footnotetext{
${ }^{3}$ Here, notice that the range of $\epsilon$ allowing the presence of the optimum solution is determined under either $\epsilon \in\left(0, \mathrm{e}^{-N_{l} \mathrm{e}}\right]$ or $\epsilon \in\left(\mathrm{e}^{-N_{l} \mathrm{e}}, 1\right]$ for a given $N_{l}$, which is introduced in Section VI.
} 


$$
n_{l}^{o p t} \cong \begin{cases}\arg \min _{n_{l}}\left|\log (-\log \epsilon)-c-\left(\log n_{l}-n_{l}^{-1}\left(\bar{n}+\left(\eta N_{l}\right)^{-1}\right)\right)\right|, & \text { when } \epsilon \in\left(0, \mathrm{e}^{-N_{l} \cdot \mathrm{e}}\right] \\ 0, & \text { when } \epsilon=0\end{cases}
$$

3GPP deployment parameters. On this layout, the co-channel deployment of $L \in\{8,16\}$ femtocells is considered, where each is randomly deployed and intends to access the radio spectrum assigned to the macrocell.

We assume that at each femtocell, $N_{l} \in\{2,4,10\}$ FUEs are available. For given $m$ inactive channels of the macrocell, $\bar{n}=m / \sum_{l} N_{l}$ sub-channels are allocated to each FUE. Here, the ratio of the energy usage between control and data planes at each sub-channel is fixed to $\eta=3 / 4$ that is referred to the case when transmitting 3 OFDM symbols of the control and 4 OFDM symbols of the data within every frame in 3GPP LTE Femtocells [33]. Due to the downlink communications between $L$ femtocells and their FUEs, the incumbent macrocell user deployed near the femtocells should experience the interference from the femtocells no greater than $I_{o}=-30 \mathrm{~dB}$.

In Fig. 2, the average interference at the incumbent receiver by multiple co-channel femtocells has been depicted with respect to the number of available sub-channels at various values of the number of femtocell users per femtocell. For this illustration, we use that for given $L=16$ femtocells deployment, each femtocell has the same number of $N_{l}$ femtocell users at different values of $N_{l} \in\{2,4,10\}$ and the proposed power selection scheme per femtocell is exploited. For comparison, on the basis of water-filling, the (conventional) greedy approach for a given average power level has also been illustrated (which is referred to as the greedy scheme hereinafter). As can be seen in this figure, when the number of $\bar{n}$ available sub-channels increases, the interference caused by the proposed power selection scheme remains at the threshold $\left(I_{o}=-30 \mathrm{~dB}\right)$ for all $N_{l}$ 's, while the interference resulting from the greedy schemes increases with $\bar{n}$ higher than the threshold for a given $N_{l}$.

It can be shown in Fig. 3 that the proposed power allocation level per femtocell normalized by that for the greedy scheme is depicted with respect to the number of available sub-channels. It can be clearly seen in this figure that as compared to the greedy scheme, the resulting proposed power allocation level is a decreasing function of $\bar{n}$ for given $L, N_{l}, \eta$, and $I_{o}$. For example, as compared to the greedy scheme with the conventionally fixed average power level, it can be observed in this figure that for a given $\bar{n}=10$, the proposed scheme with $N_{l}=2$ FUEs per femtocell only needs a power consumption at about 55 per-cent of what the greedy scheme does in order to satisfy the interference requirement.

Using the above proposed power selection scheme, it has now been illustrated in Fig. 4 that when the number of active sub-channels is given to its maximum $\bar{n}$, the outage capacity of a certain femtocell-of-interest (i.e., femtocell $l$ ) is a decreasing function of $\bar{n}$. For this illustration, we use that the several target outage probabilities $\epsilon \in\left\{10^{-1}, 10^{-2}\right\}$, $N_{l}=4$, the degrees of freedom per sub-channel is $k=4$, and $\bar{\rho}=5 \mathrm{~dB}$. From this figure, it is worth mentioning that as $\bar{n}$ increases, the resulting outage capacity with a fixed $n_{l}=\bar{n}$ is

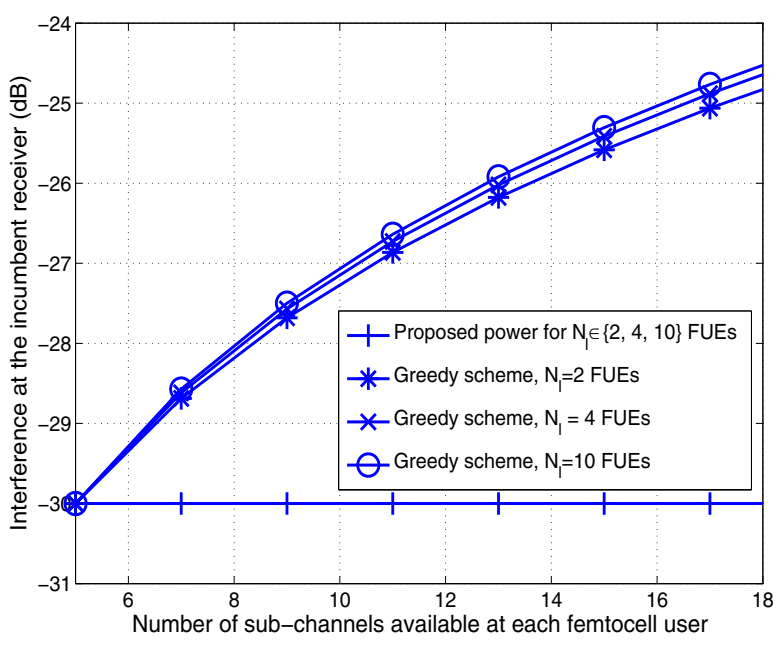

Fig. 2. Average interference at the incumbent receiver from co-channel femtocells has been illustrated with respect to the number of sub-channels available in the two cases of using greedy and proposed power selection when $L=16$ femtocells, $\eta=3 / 4, I_{o}=-30 \mathrm{~dB}, N_{l} \in\{2,4,10\} \forall l$.

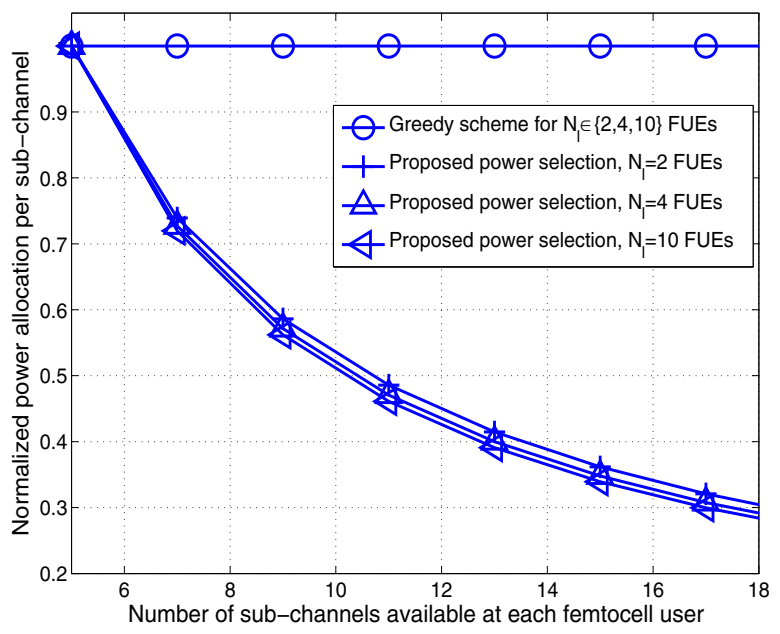

Fig. 3. Impact of the interference requirement on the power allocation to sub-channels at femtocells: When satisfying the interference requirement, the proposed power allocation to sub-channels normalized by that of the greedy power allocation has been illustrated with respect to the number of subchannels available. We used that $L=16$ femtocells, $\eta=3 / 4, I_{o}=-30 \mathrm{~dB}$, and $N_{l} \in\{2,4,10\} \forall l$.

traded off for the satisfaction of the interference requirement which validates our analysis in Section V. In addition, notice that the outage capacity is shown in this figure to increase at higher $\epsilon$ for a given $\bar{n}$.

Unlike the cases in Fig. 4, when activating only a subset of $n_{l}$ sub-channels, the outage capacity of the femtocell $l$ has been depicted in Fig. 5 with respect to the number of available sub-channels. We use that the size of a subset of active subchannels is fixed to $n_{l}$ for $n_{l} \in\{1,2,4,6\}, \epsilon=10^{-2}, N_{l}=6$, 


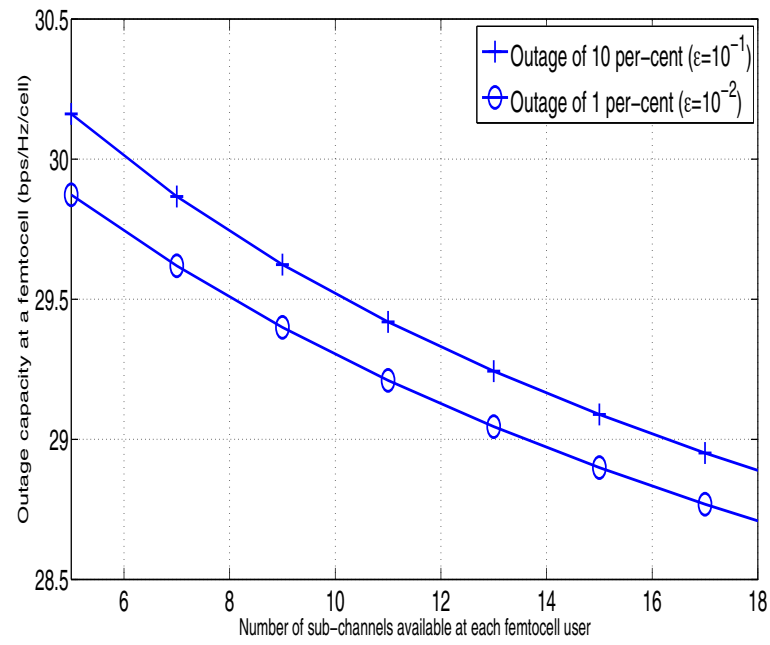

Fig. 4. Outage capacity at a femtocell versus the number of sub-channels available at various values of the target outage probability $(\epsilon)$ while the proposed power selection satisfies the interference requirement. For these illustrations, we use that $\eta=3 / 4, \epsilon \in\left\{10^{-1}, 10^{-2}\right\}, k=4, I_{o}=-30 \mathrm{~dB}$, $L=16$ femtocells, each having $N_{l}=4$ FUEs, $\forall l$ as well as a fixed $n_{l}=\bar{n}, \bar{\rho}_{l}=5 \mathrm{~dB}$.

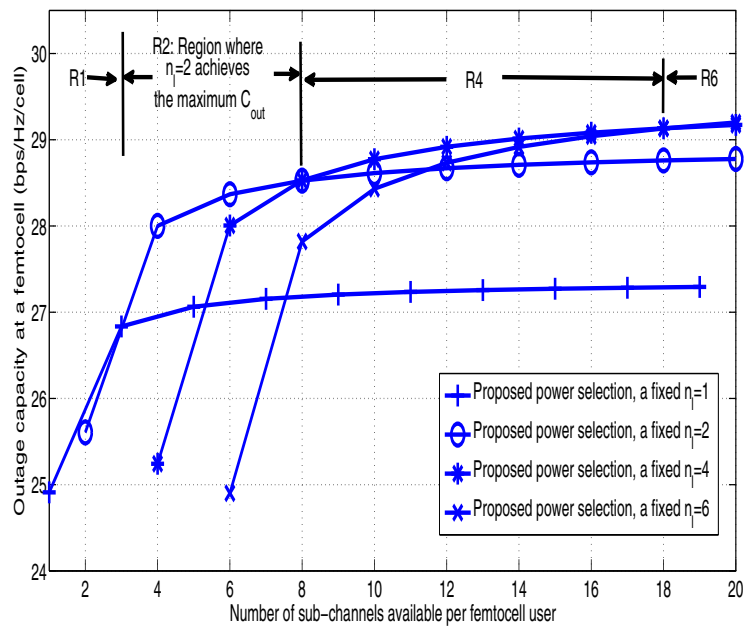

Fig. 5. Impact of activating a subset of all available sub-channels on the outage capacity: Illustration of discrete opportunistic regions for the most promising outage capacity support at a femtocell versus the number of subchannels available with various values of a fixed $n_{l} \leq \bar{n}: \eta=3 / 4, \epsilon=$ $10^{-2}, k=4, I_{o}=-30 \mathrm{~dB}, L=8$ femtocells, each having $N_{l}=6$ FUEs, $\forall l$ as well as $\bar{\rho}_{l}=0 \mathrm{~dB}$ are used.

$k=4, I_{o}=-30 \mathrm{~dB}, L=8$, and $\bar{\rho}=0 \mathrm{~dB}$. Interestingly, as seen in Fig. 5, the outage capacity is an increasing function of $\bar{n}$ by taking into account $n_{l}<\bar{n}$. In addition, for several values of $n_{l}$, it can be depicted in this figure that there exist discrete regions in terms of values of $\bar{n}$, each region consisting of a range of values of $\bar{n}$ 's under which the most promising outage capacity is achievable by the same value of $n_{l}$. For example, in a region having $\bar{n} \in\{3, \cdots, 8\}$, the outage capacity is shown in Fig. 5 to be maximized by $n_{l}=2$.

In both Figs. 6 and 7, we now illustrate the cases of using the optimum size of a subset of active sub-channels with respect to the number of available sub-channels. For

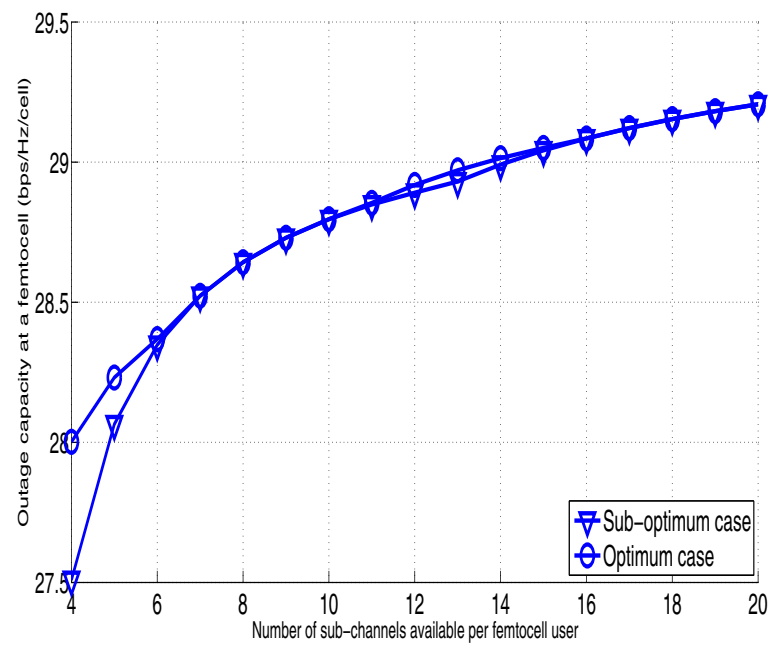

Fig. 6. Outate capacity exploiting either the optimum or the sub-optimum sizes of active sub-channels has been depicted with respect to the number of sub-channels available. For this illustration, we use that $\eta=3 / 4, \epsilon=$ $10^{-2}, k=4, I_{o}=-30 \mathrm{~dB}, L=8$ femtocells, each having $N_{l}=6$ FUEs, $\forall l$ as well as $\bar{\rho}_{l}=0 \mathrm{~dB}$.

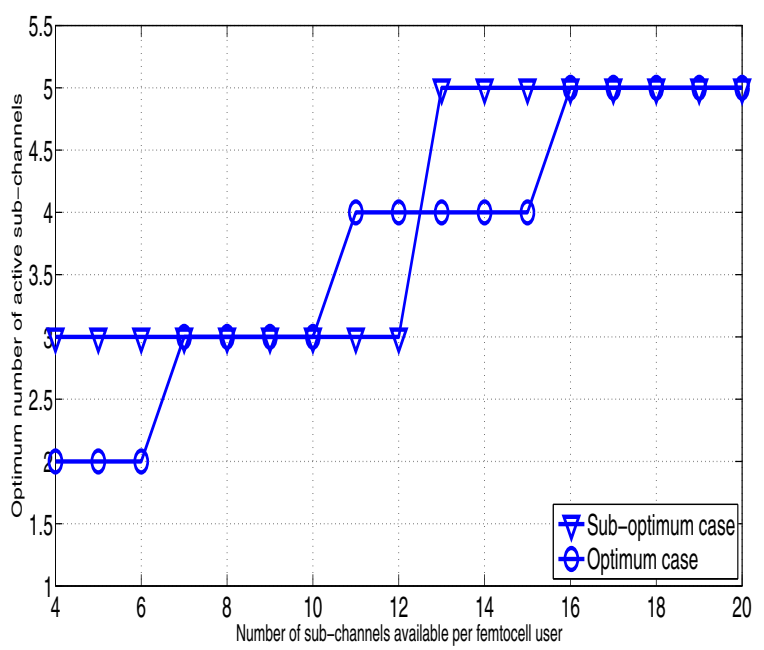

Fig. 7. Comparison of optimum and sub-optimum sets of discrete regions, each set achieving optimum and sub-optimum sizes of active subchannels, with respect to the number of sub-channels available. We use that $\eta=3 / 4, \epsilon=10^{-2}, k=4, I_{o}=-30 \mathrm{~dB}, L=8$ femtocells, each having $N_{l}=6$ FUEs, $\forall l$ as well as $\bar{\rho}_{l}=0 \mathrm{~dB}$.

comparison, a sub-optimum case has also been concerned and is depicted in both the figures. Particularly, for given values of $\bar{n}$, the optimum case takes into account all the possible values of $n_{l}$ to optimize, i.e., $n_{l} \in\{1, \cdots, \bar{n}\}$, while the suboptimum case does only a subset of all the values of $n_{l}$, i.e., $n_{l} \in\{1,2,4,6\}$. Here are two main observations to highlight. As per Fig. 6, the optimum case always outperforms the suboptimum case in terms of the outage capacity. However, as seen in Fig. 7, the sub-optimum case benefits from the less size of the set of discrete regions achieved, as compared to the optimum case. 


\section{CONCLUSION}

We considered the power resource allocation and admission control scheme for networked co-channel femtocells, over-laid to a macrocell. It was aimed at investigating the energy usage distribution among the femtocells as well as the energy usage balance between the control and the data planes per femtocell. Designing the practical energy usage model at the networked co-channel femtocells, the proper selection scheme of both the power levels at the femtocells and the size of the subset of active channels per femtocell was proposed. In the worst case of interference to the macrocell user, the performance of the proposed system has been analyzed and the closed-form expression for the asymptotic outage capacity has been derived under the outage- and the interference-stringent constraints. As per the asymptotic analysis and the numerical results, it can be clearly found from this work that in the indoor dense femtocell deployment,

- Enhanced outage capacity is achievable by jointly utilizing the size of the active subset of the incumbent subchannels and their energy usage.

- Optimum energy utilizing scheduling in the sense of the maximization of the outage capacity behaves towards the round-robin scheduling under the extremely outagestringent constraint.

Such benefits are achievable, in comparison to the conventional scheme that does not involve smart energy usage in the networked femtocells.

\section{APPENDIX A}

\section{PROOFS OF PROPOSITIONS 1 AND 2}

In order to prove Proposition 1, we first introduce the following lemma.

Lemma 1: As $x \rightarrow \infty$, let $f(x)$ and $g(x)$ tend to infinity, and $g(x)=\mathrm{o}(f(x))$. Then, we have

$$
\ln (f(x)+g(x))=\ln f(x)-\mathrm{o}(\ln g(x)) .
$$

Proof: Changing the order of the terms in (33), we need to show that

$$
\ln (f(x)+g(x))-\ln f(x)=\mathrm{o}(\ln g(x)) .
$$

This lemma is proved by noticing that

$$
\begin{aligned}
\lim _{x \rightarrow \infty} \frac{\ln (f(x)+g(x))-\ln f(x)}{\ln g(x)} & =\lim _{x \rightarrow \infty} \frac{\ln (1+g(x) / f(x))}{\ln g(x)} \\
& =0 .
\end{aligned}
$$

This lemma is now proved.

Using the above lemma, we now prove Proposition 1.

Proof: Notice that as shown in [30], $\Gamma(k, x)$ can be approximated as

$$
\lim _{x \rightarrow \infty} \Gamma(k, x)=\lim _{x \rightarrow \infty}(k-1) ! \mathrm{e}^{-x} \sum_{j=0}^{k-1} x^{j} / j !=\mathrm{e}^{-x} x^{k-1} .
$$

Applying $F_{x}(x)$ and (36) to the definition of $a_{m_{l}}$ (i.e., $a_{m_{l}}=F_{x}^{-1}\left(1-1 / m_{l}\right)$ shown above (21)), we have $\lim _{m_{l} \rightarrow \infty} F_{x}\left(a_{m_{l}}\right)=1-\frac{\mathrm{e}^{-a_{m_{l}} a_{m_{l}}{ }^{k-1}}}{\Gamma(k)}=1-1 / m_{l}$. From this, it can be obtained for large $m_{l}$ that

$$
\mathrm{e}^{-a_{m_{l}}} a_{m_{l}}{ }^{k-1}=\Gamma(k) / m_{l} .
$$

By taking logarithm of both sides of (37), $a_{m_{l}}$ can be expressed as

$$
a_{m_{l}}=\ln m_{l}-\ln \Gamma(k)+(k-1) \ln a_{m_{l}} .
$$

Since $a_{m_{l}}=\mathrm{o}\left(\mathrm{m}_{\mathrm{l}}\right)$ or, equivalently, $\ln a_{m_{l}}=\mathrm{o}\left(\ln \mathrm{m}_{\mathrm{l}}\right)$, using Lemma $1, \ln a_{m_{l}}$ in (38) is given by

$$
\begin{aligned}
\ln a_{m_{l}} & =\ln \left(\ln m_{l}-\ln \Gamma(k)+(k-1) \ln a_{m_{l}}\right) \\
& =\ln \left(\ln m_{l}\right)+\mathrm{o}\left(\ln \ln m_{l}\right) .
\end{aligned}
$$

Substituting (39) into (38), we obtain

$$
\begin{aligned}
a_{m_{l}} & =\ln \frac{m_{l}}{\Gamma(k)}+\ln \left(\ln m_{l}-\ln \Gamma(k)+(k-1) \ln a_{m_{l}}\right)^{(k-1)} \\
& =\ln \frac{m_{l}}{\Gamma(k)}+\ln \left(\ln m_{l}+(k-1) \ln \ln m_{l}\right)^{(k-1)} \\
& +\mathrm{o}\left(\ln \ln \ln m_{l}\right) .
\end{aligned}
$$

For $b_{m_{l}}$, notice that $b_{m_{l}}=a_{\mathrm{em}_{1}}-a_{m_{l}}$ [31]. Thus, a similar train of arguments as for $a_{m_{l}}$ can easily be applied.

We now prove Proposition 2.

Proof: Let the argument of $G_{y}(\cdot)$ in (22) be denoted by $A$ for simple description here. Referring to (21), then, taking a natural logarithm on both sides of (22) will produce

$$
\log G_{y}(A) \leq \log \epsilon \Rightarrow \exp (-A) \geq-\log \epsilon .
$$

By taking another natural logarithm on both sides of this expression, we have

$$
A \leq-\log (-\log \epsilon) .
$$

Here, note that the notation $A$ is the argument of $G_{y}(\cdot)$ in (22) and thus, after simple mathematical manipulations, we achieve

$$
\begin{aligned}
2^{C_{\text {out }, l}-1} \leq & \bar{\rho}_{l} P_{c}\left(N_{l}\left(\bar{n}-n_{l}\right)+\eta^{-1}\right)^{-1} \\
& \times\left(a_{m_{l}}-b_{m_{l}} \log (-\log \epsilon)\right) .
\end{aligned}
$$

From this, obtaining (25) is straightforward.

\section{ACKNOWLEDGMENT}

The research leading to these results was derived from the European Communitys Seventh Framework Programme (FP7) under Grant Agreement number 248454 (QoSMOS).

\section{REFERENCES}

[1] V. Chandrasekhar, J. Andrews, and A. Gatherer, "Femtocell networks: a survey," IEEE Commun. Mag., vol. 46, no. 9, pp. 59-67, Sep. 2008.

[2] H. Claussen, "An overview of the femtocell concept," Bell Labs Tech. J., 2008.

[3] J. Zhang and G. Roche, Femtocells: Technologies and Deployment, 1st edition. Wiley, 2010.

[4] V. Chandrasekhar and J. Andrews, "Uplink capacity and interference avoidance for two-tier femtocell networks," IEEE Trans. Wireless Commun., vol. 8, no. 7, pp. 3498-3509, July 2009.

[5] D. Lopez-Perez, G. de la Roche, A. Valcarce, A. Juttner, and J. Zhang, "Interference avoidance and dynamic frequency planning for WiMAX femtocells networks," in Proc. 2008 IEEE Intr. Conf. Commun. Syst., pp. 1579-1584. 
[6] X. Chu, J. Y. Wu, and H. Wang, "Outage probability analysis for collocated spectrum-sharing macrocell and femtocells," in Proc. 2011 IEEE Intr. Conf. Commun. Syst., pp. 1-5.

[7] J. S. Wu, J. K. Chung, and M. T. Sze, "Analysis of uplink and downlink capacities for two-tier cellular system," IEE Proc. Commun., vol. 144, no. 6, pp. 405-411, Dec. 1997.

[8] R. S. Karlsson, "Radio resource sharing and capacity of some multiple access methods in hierarchical cell structures," in Proc. 1999 IEEE Veh. Technol. Conf. - Fall, vol. 5, pp. 2825-2829.

[9] The 3rd Generation Partnership Project TS 25.469, "UTRAN Iuh interface Home Node B (HNB) application part (HNBAP) signalling (release 9)," vol. 9.2.0, June 2010.

[10] The 3rd Generation Partnership Project TS 36.921, "Home eNB RF requirements analysis (release 9)," vol. 9.1.0, Mar. 2010.

[11] C. Lima, M. Bennis, K. Ghaboosi, and M. Latva-aho, "On interference analysis of self-organized femtocells in indoor deployment," in Proc. 2010 IEEE Globecom, pp. 659-663.

[12] F. Pantisano, M. Bennis, R. Verdone, and M. Latva-aho, "Interference management in femtocell networks using distributed opportunistic cooperation," in Proc. 2011 IEEE Veh. Technol. Conf. - Spring, pp. 1-5.

[13] D. Lopez-Perez, A. Valcarce, G. de la Roche, and J. Zhang, "OFDMA femtocells: a roadmap on interference avoidance," IEEE Commun. Mag., vol. 46, no. 9, pp. 59-67, June 2009.

[14] V. Chandrasekhar, J. G. Andrews, T. Muharemovic, Z. Shen, and A. Gatherer, "Power control in two-tier femtocell networks," IEEE Trans. Wireless Commun., vol. 8, no. 8, pp. 4316-4328, Aug. 2009.

[15] L. T. W. Ho and H. Claussen, "Effects of user-deployed, co-channel femtocells on the call drop probability in a residential scenario," in Proc. 2007 IEEE International Symp. Personal, Indoor Mobile Radio Commun., pp. 1-5.

[16] H. Claussen, "Performance of macro- and co-channel femtocells in a hierarchical cell structure," in Proc. 2007 IEEE International Symp. Personal, Indoor Mobile Radio Commun., pp. 1-5.

[17] Y. Kim, S. Lee, and D. Hong, "Performance analysis of two-tier femtocell networks with outage constraints," IEEE Trans. Wireless Commun., vol. 9, no. 9, pp. 2695-2700, Sep. 2010.

[18] H.-S. Jo, C. Mun, J. Moon, and J.-G. Yook, "Interference mitigation using uplink power control for two-tier femtocell networks," IEEE Trans. Wireless Commun., vol. 8, no. 10, pp. 4906-4910, Oct. 2009.

[19] Y. Hou and D. I. Laurenson, "Energy efficiency of high QoS heterogeneous wireless communication network," in Proc. 2010 IEEE Veh. Technol. Conf. - Fall, pp. 1-5.

[20] I. Ashraf, L. T. W. Ho, and H. Claussen, "Improving energy efficiency of femtocell base stations via user activity detection," in Proc. 2010 IEEE Wireless Commun. Netw. Conf., pp. 1-5.

[21] The 3rd Generation Partnership Project TR 36.814, "Evolved universal terrestrial radio access (E-UTRA); further advancements for E-UTRA physical layer aspects (release 9)," vol. 9.0.0, Mar. 2010.

[22] V. Chavali and C. da Silva, "Collaborative spectrum sensing based on a new SNR estimation and energy combining method," IEEE Trans. Veh. Technol., vol. 60, no. 8, pp. 4024-4029, Oct. 2011.

[23] D. Godarzi, K. Arshad, Y. Ko, and K. Moessner, "Selecting users in energy-efficient collaborative spectrum sensing," in Proc. 2012 IEEE Wireless Commun. Netw. Conf., pp. 1029-1033.

[24] Q. Ma and C. Tepedelenlioğlu, "Practical multiuser diversity with outdated channel feedback," IEEE Trans. Veh. Technol., vol. 54, pp. 1334-1345, July 2005.
[25] D. Tse and P. Viswanath, Fundamentals of Wireless Communication, 1st edition. Cambridge University Press, 2005.

[26] R. Knopp and P. A. Humblet, "Information capacity and power control in single-cell multiuser communications," in Proc. 1995 IEEE Intl. Conf. Commun., vol. 1, pp. 331-335.

[27] Y. Ko, S. A. Vorobyov, and M. Ardakani, "How much multiuser diversity is required for energy-limited multiuser systems?" IEEE Trans. Signal Process., vol. 58, no. 8, pp. 4367-4378, Aug. 2010.

[28] A. Doufexi, S. Armour, M. Butler, A. Nix, D. Bull, J. McGeehan, and P. Karlsson, "A comparison of the HIPERLAN/2 and IEEE 802.11a wireless LAN standards," IEEE Commun. Mag., vol. 40, no. 5, pp. 172180, May 2002.

[29] B. Ghimire, G. Auer, and H. Haas, "Busy bursts for trading off throughput and fairness in cellular OFDMA-TDD," EURASIP J. Wireless Commun. Netw., vol. 2009 (Article ID 462396), 2009.

[30] I. S. Gradshteyn and I. M. Ryzhik, Table of Integrals, Series, and Products, 6th edition. Academic Press, 2000.

[31] E. Castillo, Extreme Value Theory in Engineering. Academic Press, 1988.

[32] B. M. Hochwald, T. L. Marzetta, and V. Tarokh, "Multiple-antenna channel hardening and its implications for rate feedback and scheduling," IEEE Trans. Inf. Theory, vol. 50, pp. 1893-1909, 2004.

[33] The 3rd Generation Partnership Project TS 36.211, "Evolved universal terrestrial radio access (E-UTRAN); physical channels and modulation (release 9)," vol. 9.1.0, Mar. 2010. 